retrospective review of these attendances looked for three key performance indicators, NTproBNP assessment, echocardiogram and referral to heart failure/cardiology service as good quality care.

Results There were 227 attendances to the emergency department during the six months of the study period. 186 patients were admitted and 41 patients were discharged home. One patient visited the emergency department on 3 occasions therefore 39 patients attended the emergency department. The average age was 72.7 years with $59 \%$ male. A new diagnosis was established in $56 \%$ (22) with a pre existing diagnosis in the others. After the emergency department attendance 49\% (19) had no follow up arranged. In 64\% (25) no NTproBNP was undertaken. an echocardiogram was ordered for $28 \%$ (11). Patients not requiring an echocardiogram due to low NTproBNP or previous echo in last 6 months 30\% (12). In $23 \%$ (9) none of the key indicators were undertaken; no NTproBNP, echocardiogram or follow up.

Conclusion Nearly a quarter of patients 23\% (9) were discharge from the emergency department with a diagnosis of heart failure and did not receive any of the performance indicators of NTproBNP, echocardiogram or follow up. It is unsure if these patients do actually have heart failure and potentially may have been started on inappropriate medication or if they indeed do have heart failure and are not treated appropriately with the impact of increased mortality and further readmissions. This is a gap that has been identified in our local heart failure service provision and suspect that such a provision gap may be widespread in other areas. A redesign of our service is intended to address this gap.

Conflict of Interest None

\section{A NOVEL ULTRASOUND BASED METHOD FOR SCREENING FOR HEART FAILURE}

Anenta Ramakrishnan, Ryan Reavette, Ethan Rowland, Jamil Mayet, Peter Weinberg. Imperial College London, London, UK

\subsection{6/heartjnl-2021-BCS.137}

Introduction The ventricles accelerate and decelerate blood; the resulting disturbances propagate through the arterial system as waves. The magnitude and timing of these waves vary with cardiac performance and their speed depends on arterial stiffness. These properties can be studied by using 'Wave Intensity Analysis' (WIA), which is a method that describes the energies that drive arterial flow as distinct 'waves'. WIA has been shown to be altered in heart failure with reduced ejection fraction (HFrEF)and thus has diagnostic potential. Routine clinical application of WIA has been limited, because conventional WIA relies on invasive catheter measurements of blood pressure and velocity. However, we have developed and validated a new non-invasive ultrasound-based method for WIA. We have previously shown in-vivo that this method allows accurate WIA compared to invasive catheter measurements in rabbits.

Aim We aimed to evaluate the feasibility of using this novel non-invasive WIA technology to predict heart failure with reduced ejection fraction.

Methods Patients attending outpatient Cardiology clinics and their accompanying friends and family were invited to participate. Diameter and velocity were simultaneously measured using an ultrafast ultrasound imaging system (Verasonics,
Kirkland, USA). Blood signal was enhanced using spatio-temporal filtering. After applying standard cross-correlation techniques, a novel WIA formulation based upon diameter and velocity was calculated. Three ultrasound measurements of six seconds each were taken at each of the left carotid, right carotid and left or right brachial arteries. Post-processing and analysis were performed using custom-written software (Matlab). Heart failure was assessed by clinical evaluation. Echocardiography was performed by a trained echocardiographer to the British Society of Echocardiography guidelines within 1 year of the ultrasound measurements for all heart failure patients and for most non-heart failure patients.

Results 109 participants were recruited with written consent. WIA has been calculated for 45 participants to date. Preliminary analysis has shown significant differences in WIA metrics between participants with HFrEF $(n=19)$ and participants without failure $(\mathrm{n}=26)$. Specifically, the ' $\mathrm{S}$ ' wave component of WIA corresponds to the energy that is generated by the left ventricular and responsible for acceleration of flow in systole. Our preliminary analysis shows that the ' $\mathrm{S}$ ' wave was reduced in participants with heart failure with reduced ejection fraction compared to participants without heart failure.

Conclusion This novel ultrasound-based technology enables wave intensity analysis measurements to be obtained non-invasively at the carotid or brachial artery. WIA measured using this novel ultrasound-based technology could provide a clinically useful way to detect heart failure with reduced ejection fraction (HFrEF).

Conflict of Interest none

\section{PATIENT FLOW THROUGH A SPECIALIST HEART FAILURE CLINIC: A TIME AND MOTION STUDY}

${ }^{1}$ Arvind Singhal, ${ }^{1}$ Serena Ng, ${ }^{2}$ Martin Cowie. ${ }^{1}$ Royal Brompton Hospital, London, UK; ${ }^{2}$ Imperial College, London

\subsection{6/heartjnl-2021-BCS.138}

Introduction Heart Failure (HF) is a chronic disease requiring regular monitoring and follow-up. Efficient utilisation of $\mathrm{HF}$ clinic time is essential for both HF patients and overburdened healthcare systems. We used time-and-motion studies, examining when (time) and how (motion) processes occur, in HF clinics at a specialist centre to identify areas for improved efficiency. Methods Time-and-motion studies were conducted for four consultant-led HF clinics between 3rd December 2019 and 18th February 2020. Investigators noted the time of arrival and departure of each patient in the following clinical areas: electrocardiography (ECG), echocardiography, pacing department, consultation room, phlebotomy and x-ray. Estimated journey times for patients were calculated using Google Maps. Results 60 patients attended appointments, and complete data were collected for 58 patients. The mean age was 66.6 years (SD \pm 13.2 ), and $31 \%$ of patients were female. Patients spent a median of 103 minutes in the hospital (IQR 76 - 137 mins). Consultations were a median of 20 minutes long (IQR 14 27 mins), a median of 23 minutes after their scheduled time (IQR $6-74$ mins) and a median of 63 minutes after patients arrived (IQR 34 to 93.5 mins). Figure 1 is a box plot showing the time patients spent undergoing each investigation on the same day as their appointment, alongside time spent in consultation. Blood tests and ECGs were the most common investigations coinciding with the hospital visit, performed 